\section{Bases científicas del razonamiento clínico: inferencia Bayesiana}

\author{
MAURICIO CANALS L. ${ }^{1}$
}

\section{Bayesian inference in clinical reasoning}

A conceptual analysis of diagnostic reasoning in clinical practice is carried out. Using Bayesian inference as an alternative to frequentist inference usually used in science, clinical reasoning uses the scientific method step by step. The concepts of scientific method, probability, statistics and Bayesian inference are reviewed, highlighting their fundamental differences with the frequentist approach. This paper shows how the diagnostic approach proceeds in a Bayesian sense, ending with a basic example of application.

(Rev Med Chile 2019; 147: 231-237)

Key words: Bayes Theorem; Science; Statistics.

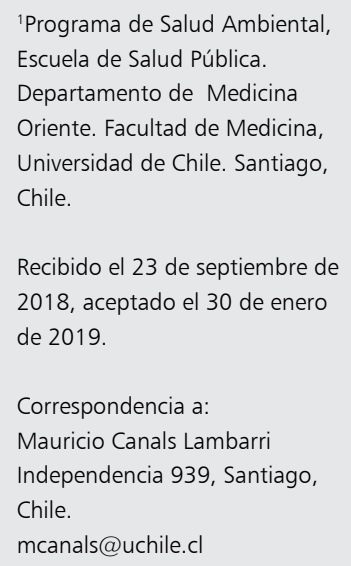

U n respetado profesor de mis primeros años de estudios de medicina incluía una sencilla pregunta de tres alternativas: " $\mathrm{La}$ medicina es: a) un arte; b) una ciencia y c) una ciencia y un arte". La respuesta era "c". Pasado los años, pienso que este profesor quería enseñarnos que la medicina se sustenta en una fuerte base científica, pero que la práctica médica consta de muchos otros aspectos como lenguaje, habilidades de comunicación y relaciones humanas, que no pueden ser enmarcados en el método científico.

Actualmente se ha propuesto que en el proceso diagnóstico intervienen dos aproximaciones: una rápida, intuitiva que requiere mínimos esfuerzos cognitivos que podríamos analogar al "ojo clínico" y la otra lenta, analítica y deliberada que requiere un mayor esfuerzo consciente ${ }^{1,2}$, siendo discutido cuál es más efectiva ${ }^{3-6}$. Para muchos médicos, hoy este "arte" de diagnosticar es sólo cuestión de "saber qué hacer en cada situación". Sin embargo, los problemas que presentan los pacientes y los enfoques de diagnóstico diferencial son dependientes del contexto en que se presentan. Por ejemplo, ante la presencia de cefalea el diagnóstico diferencial y el enfoque anamnéstico desde la medicina ambulatoria y desde un servicio de urgencia son diferentes ${ }^{7}$.

Mucha de la medicina de hoy se basa en tener algoritmos de acción o normas de acción ante los problemas $^{8,9}$. Pero ¿es todo algoritmizable? Kurt Gödel en 1931, demostró que ninguna teoría matemática formal es a la vez consistente (no existen contradicciones) y completa (responde todas las preguntas). Demostró que existen hipótesis indecidibles y por tanto no es posible crear un algoritmo capaz de dar cuenta de todas las interrogantes en un cuerpo de conocimiento. Posteriormente, en 1963 Paul Cohen, demostró que siembre que hay $\mathrm{n}$ axiomas es necesario uno más $(\mathrm{n}+1)^{10}$. Así, no todo puede ir en normas o manuales y aún hay espacio para pensar.

Otra manera de ver el proceso diagnóstico es pensar que todo se trata de método para llegar a un diagnóstico adecuado. Pero ¡es este método científico?

\section{Método científico}

Gracias a la contribución de numerosos autores se fue consolidando el método hipotético-deductivo cuyos pasos fundamentales son la observación, creación de hipótesis, deducción de consecuencias verificables, la verificación y comparación con la experiencia. Descartes (15961650) definió "las reglas del método para dirigir 
bien la razón y buscar la verdad en las ciencias" y Francis Bacon (1561-1626) pensaba que la observación repetida de fenómenos comparables permitía extraer por inducción leyes generales que los gobiernan. Sin embargo, Karl Popper (1902-1994) rechazó la posibilidad de extraer leyes generales por inducción aclarando que estas constituyen en realidad nuevas hipótesis que permiten elaborar predicciones $^{11,12}$. Para Popper lo central es que las teorías puedan ser refutadas ("falsabilidad") y en este sentido no habría teorías verdaderas sino no-refutadas. El método de Popper es conocido como hipotético-deductivo-refutacionista (HDR). En esta dualidad de opiniones de Bacon y Popper parece establecerse una controversia entre el pensamiento inductivo que propone la obtención de leyes generales desde la repetitividad, desde lo particular a lo general (empírico, observacional); y el pensamiento deductivo que va desde lo general a lo particular (si p, entonces q). Sin embargo, de las cuatro fases del método HDR: 1) planteamiento del problema; 2) creación de hipótesis; 3) deducción de consecuencias verificables de la hipótesis, y 4) contraste de hipótesis (refutación), la deducción sólo participa en los pasos 2 y 3 . Mientras que la inducción opera en los pasos 1 y 4 . Así, el método científico combina inducción y deducción. Hoy algunos autores rechazan la refutación como el único sello de lo científico ${ }^{13}$.

\section{Estadística y la definición de probabilidad}

El razonamiento inductivo conduce a evaluaciones de determinadas proposiciones, y esto se trata en la estadística que se puede definir como "la ciencia, pura y aplicada, que crea, desarrolla y aplica técnicas para la descripción de datos y la evaluación de la incertidumbre de inferencias inductivas" (modificada de ref. 14). Es decir el fin de la estadística es medir o cuantificar la incerteza de las inferencias inductivas mediante el concepto de probabilidad.

La definición clásica de Laplace propone que si todos los sucesos elementales del espacio muestral son equiprobables (mutua simetría), la probabilidad del suceso A es el cociente entre el número de resultados favorables a $\mathrm{A}$ y el número de resultados posibles del experimento:

$$
P(A)=\frac{\text { casos favorables }}{\text { casos posibles }}
$$

Debemos notar dos hechos: 1) en esta definición no se necesita haber realizado el experimento, es decir es una definición a priori. No sería necesario tirar un dado para saber que la probabilidad de sacar un 5 es 1/6; y 2) La definición es circular, es decir para definir probabilidad se necesita que los sucesos elementales del espacio muestral sean equiprobables (mutua simetría). O sea, se define probabilidad en función de la equiprobabilidad del espacio muestral. Esto constituye la paradoja de la teoría de probabilidades.

Maurice Frechet en cambio planteó que es posible estimar la probabilidad del suceso A como la frecuente relativa de ocurrencia de $\mathrm{A}(n A)$ en $\mathrm{n}$ intentos $(n)$ : Esta es una definición a posteriori, es decir, exige que el experimento se haya realizado repetidas veces, antes de estimar la probabilidad de un suceso, y crea la noción frecuentista de probabilidad. Una crítica a la definición de Frechet, es que no responde a la pregunta icuantas veces hay que repetir el experimento? Esto lo que quiso corregir Richard Von Mises, definiendo la probabilidad como un límite:

$$
P(A)=\frac{\lim ^{\frac{n A}{n}}}{n \rightarrow \infty}
$$

Sin embargo, no es posible repetir un experimento infinitas veces.

Andrei Kolmogorov, consciente de la paradoja de la definición clásica de probabilidad miró la probabilidad desde la teoría de la medida y propuso una definición axiomática como una función que le asigna a un evento, un número $\mathrm{P}(\mathrm{A})$ entre 0 y 1 eludiendo el problema de la mutua simetría. Su definición es a posteriori pues para determinar la medida de la probabilidad se necesita medir el evento A y medir el espacio muestral ${ }^{15,16}$.

Vemos en estas definiciones una dualidad que se refleja en la siguiente pregunta: ¿Es la probabilidad un atributo del evento, o es un atributo del conocimiento? Las definiciones a posteriori exigen que el experimento se haya realizado, siendo entonces la probabilidad un atributo del evento. Pero si el experimento ya se realizó y conocemos el espacio muestral y su medida, ¿para que necesitamos el concepto de probabilidad? La definición clásica en cambio no requiere que el experimento se haya realizado, pero para su estimación se requiere el conocimiento de los sucesos elementales que constituyen el espacio muestral. Esta dualidad se hace 
explícita en el siguiente ejemplo. Hay dos sujetos; el sujeto 1 tira una moneda al aire, ve el resultado sin que el otro lo vea y le pregunta al sujeto 2: ¿cuál es la probabilidad que sea sello? El sujeto 2 dice inmediatamente $1 / 2$. Pero el evento ya ocurrió y entonces el sujeto 1 dice: ¡no! La probabilidad es 1, salió sello. El sujeto 1 conoce el evento y su frecuencia relativa es 1 ( 1 en 1 intento) mientras que el segundo mide su conocimiento del posible resultado $(1 / 2)$.

\section{Estrategia de la estadística frecuentista}

La ciencia se basa en datos y el rechazo o aceptación de la hipótesis requiere la medición de la incerteza de las inferencias inductivas ( $\mathrm{p}$-valor). La estrategia estadística consiste en 1) Planteamiento de la Hipótesis; 2) Dicotomía de la hipótesis: $H_{0}$ vs $H_{1}$, donde $H_{0}$ es la hipótesis de nulidad y $H_{1}$ es la hipótesis alternativa (de investigación); 3) Elección del nivel de significación $(\alpha)$. En general en ciencias se consideran como adecuados niveles menores que $\alpha=0,1 ; 0,05 ; 0,01$. El más aceptado es $\alpha=0,05$, aunque para estudios de bajo poder se han propuesto niveles de hasta $0,001^{17}$; 4) Elección de la dócima apropiada (test), basados en ciertos supuestos; y 5) En base al resultado de la dócima, tomar una decisión estadística. De esta manera, la estrategia de la estadística frecuentista va dirigida al rechazo de la hipótesis de nulidad $H_{0}$, lo que permite, dado la dicotomía de la hipótesis, aceptar por defecto la hipótesis de investigación contenida en $H_{1}$.

Para la toma de decisiones la estrategia es medir la probabilidad de rechazar $H_{0}$ dado que esta es verdadera, es decir, se estima a partir de los datos $P\left(\right.$ RechazoH $\left.H_{0} / H_{0}=V\right)=P($ Error de tipo $I)$, lo que conocemos como el p-valor. Si este es menor que el máximo nivel que estamos dispuesto a tolerar (ie $p<\alpha$ ), entonces rechazamos $H_{0}$ y en consecuencia aceptamos $H_{1}$. Observamos dos hechos realmente sorprendentes: 1) lo que medimos es en esencia la probabilidad de tener los datos observados $\left(X_{i}\right)$ bajo el supuesto que $H_{0}$ es verdadera $P\left(X_{i} / H_{0}\right)$ y no $P\left(H_{0} / X_{i}\right)$; si esta probabilidad es baja entonces es falsa; y 2) nunca probamos $H_{1}$ ni medimos su probabilidad, es decir la aceptamos por defecto.

Si aceptamos $H_{0}$ podemos estar cometiendo otro error (de tipo II), que es medible $P$ (Aceptar $\left.H_{0} / H_{0}=F\right)=P\left(\right.$ Aceptar $\left.H_{0} / H_{1}=V\right)$, pero habitualmente no lo medimos ${ }^{18}$.
La estrategia estadística fue construida por un pequeño grupo de estadísticos, la mayoría ingleses, Francis Galton, Karl Pearson, William Gosset (autodenominado Student), Ronald Fisher, Jerzy Neyman y Egon Pearson. En la estrategia de Fisher, sólo existe un error: rechazar una hipótesis de nulidad cuando es verdadera. La segunda forma de error fue introducida por Neyman y E. Pearson, llamando al error de Fisher error de tipo I y a este nuevo error, error de tipo II. Según Fisher, el nivel de significación equivale a la magnitud máxima del riesgo que está dispuesto a correr el investigador, de cometer el error de rechazar una hipótesis de nulidad cuando es verdadera. Sin embargo en la perspectiva de Neyman y Pearson, para establecer el nivel de significación estadística habría que estudiar cada tipo de error, y a partir de ahí se decidiría cuál de ellos es preferible minimizar. A partir de este último tipo de error, introdujeron el concepto de "poder de una prueba estadística", el cual se refiere a su capacidad para evitar el error tipo II, y está definido por $1-\beta$ (medida del error de tipo II), y a partir de éste se ha desarrollado el concepto de "tamaño del efecto" que algunos han propuesto como sustituto de los valores $\mathrm{p}$ en los informes de investigación científica ${ }^{19-22}$. Aquí es útil aclarar que el p-valor no mide el tamaño del efecto ni estima la verosimilitud de $H_{1}$, lo que hace que la frase "fue estadísticamente significativo" induzca a errores de interpretación, por lo que la Sociedad americana de Estadística (ASA) ha sugerido evitarla ${ }^{23}$.

Vemos que gran parte del conocimiento científico se basa en un método que tiene varias grietas conceptuales como la paradoja de las probabilidades y una estrategia al menos extraña, pero que sin embargo, aceptamos como una especie de híbrido de los conceptos de Pearson, Fisher y Neyman.

¿No habrá otro proceso inferencial que pueda solucionar estos problemas?

\section{Inferencia Bayesiana}

En 1763 Thomas Bayes enunció su famoso teorema: "Dada una partición del espacio muestral $A_{i}$ (ie: diagnósticos) y un evento $B$ (ie: una manifestación: un hecho semiológico, un examen, etc), entonces la probabilidad condicional (o a posteriori) de $A_{i}$, dado el evento $B$ (ie: la probabilidad de un diagnóstico dado $B$ ) es: 


$$
P(A i \mid B)=\frac{P(B \mid A i) P(A i)}{\sum_{j} P(B \mid A j) P(A j)}
$$

Nos indica que para estimar (o actualizar) la probabilidad de un evento es necesario estimar la probabilidad $a$ priori de $\left(P\left(A_{j}\right)\right)$ y la probabilidad de que ocurra el evento $B$ dado cada uno de los posibles $A_{j}^{24,25}$.

$\mathrm{Al}$ extender este teorema a variables aleatorias continuas y a la estimación de parámetros $(\theta)$ a partir de los datos $\left(X_{i}\right)$ podemos establecer que la probabilidad a posteriori dado el conjunto de datos $\left(\varpi\left(\theta \mid X_{i}\right)\right)$ es proporcional a la verosimilitud de los datos $\left(P\left(X_{i} \mid \theta\right)=l(\theta)\right)$ ( por la probabilidad a priori $\varpi(\theta))$.

Este teorema permite medir directamente la verosimilitud de una hipótesis de investigación. Analicemos el siguiente desarrollo. Sólo son posibles $H_{0}$ y $H_{1}$ y queremos medir directamente la probabilidad que $H_{1}$ sea verdadera habiendo obtenido un conjunto de datos $X_{i}$. Aplicando directamente el teorema de Bayes:

$$
\begin{gathered}
P\left(H_{1} \mid X_{i}\right)=\frac{P\left(X_{i} \mid H_{1}\right) P\left(H_{1}\right)}{P\left(X_{i} \mid H_{1}\right) P\left(H_{1}\right)+P\left(X_{i} \mid H_{0}\right) P\left(H_{0}\right)}=\frac{P\left(X_{i} \mid H_{1}\right) P\left(H_{1}\right)}{P\left(X_{i}\right)} \\
\mathrm{Y} \\
P\left(H_{0} \mid X_{i}\right)=\frac{P\left(X_{i} \mid H_{0}\right) P\left(H_{0}\right)}{P\left(X_{i} \mid H_{1}\right) P\left(H_{1}\right)+P\left(X_{i} \mid H_{0}\right) P\left(H_{0}\right)}=\frac{P\left(X_{i} \mid H_{0}\right) P\left(H_{0}\right)}{P\left(X_{i}\right)}
\end{gathered}
$$

Dividiendo ambas obtenemos:

$$
\frac{P\left(H_{1} \mid X_{i}\right)}{P\left(H_{0} \mid X_{i}\right)}=\frac{P\left(X_{i} \mid H_{1}\right)}{P\left(X_{i} \mid H_{0}\right)} \frac{P\left(H_{1}\right)}{P\left(H_{0}\right)}
$$

Esta razón es el factor de Bayes de $H_{1}$ y mide cuanto más probable es $H_{1}$ que $H_{0}$, dado los datos, es decir la Odds a posteriori de $H_{1}\left(\mathrm{OpH}_{1}\right)^{26,27}$. Su inverso es la Odds a posteriori de $H_{0}$.

Dos observaciones: 1) conociendo la Odds a posteriori de $H_{1}$ podemos calcular su probabilidad $P\left(H_{1} / X_{i}\right)=\mathrm{OpH}_{1} /\left(\mathrm{OpH}_{1}+1\right)$ y 2$)$ este es un resultado habitual muy usado en medicina basada en la evidencia: La Odds a posteriori = razón de verosimilitudes x Odds a priori: $O p=L R x O_{0}^{28}$.

La inferencia Bayesiana en general se realiza por métodos iterativos que permiten actualizar la probabilidad de una hipótesis dado los datos (Figura 1). Tiene la particularidad de que necesita una estimación a priori de las probabilidades de las hipótesis que puede ser subjetiva. Al igual que en la estadística frecuentista, a mayor cantidad de datos menor es la incerteza de las inferencias. Usa el concepto de probabilidad a priori como atributo del conocimiento y mide directamente la verosimilitud de la hipótesis de investigación ${ }^{29}$ (Tabla 1).

\section{Aproximación diagnóstica}

A estas alturas, es bueno preguntarse ¿Qué tiene que ver esto con el razonamiento clínico? Como se ha vislumbrado en los párrafos anteriores y como ha hecho evidente el desarrollo de la medicina basada en la evidencia ${ }^{28}$ en la práctica clínica se cumple con todo el proceso del método científico. Se conoce un problema, se plantea una hipótesis diagnóstica y se docima acumulando un conjunto de manifestaciones (datos; $X_{i}$ ) constituidos por datos de la anamnesis, signos y síntomas en el examen físico, exámenes de laboratorio, imágenes etc, para finalmente inferir una hipótesis diagnóstica. Este razonamiento diagnóstico corresponde exactamente al proceso de inferencia Bayesiana. Al igual que en este, se tienen probabilidades a priori que se actualizan en función de un conjunto de datos. En términos simples, si queremos conocer la probabilidad de un determinado diagnóstico (o su Odds) es necesario primero conocer la epidemiología (perfil de riesgo) que representa la realidad

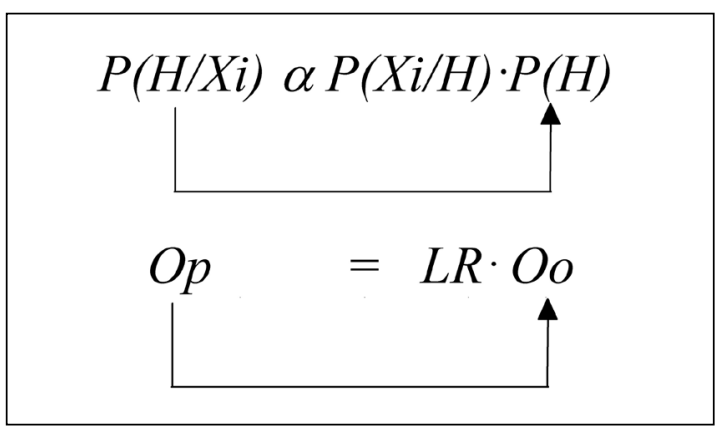

Figura 1. Inferencia Bayesiana. Arriba: El proceso consiste en la actualización de la probabilidad de una hipótesis $(P(H))$ para obtener una nueva probabilidad a posteriori dado un conjunto de datos $(P(H / X))$ ). Esta probabilidad es proporcional al producto de la verosimilitud $(P(X i / H))$ y $P(H)$. La nueva probabilidad obtenida puede ser usada como una probabilidad a priori ante un nuevo dato o conjunto de datos, dando origen a un proceso inferencial iterativo. Abajo: en forma equivalente se puede actualizar la Odds a priori $\left(O_{0}\right)$ multiplicándola por la razón de verosimilitudes $(L R)$, obteniendo el factor de Bayes en favor de la hipótesis $\mathrm{H}$, u Odds a posteriori, y continuar con el proceso iterativo. 
Tabla 1. Diferencias en la aproximación frecuentista y Bayesiana de la inferencia estadística (modificada de Ellison ${ }^{29}$ )

\begin{tabular}{|lll|}
\hline Concepto & Inferencia frecuentista & Inferencia Bayesiana \\
\hline Probabilidad & Frecuencia en gran cantidad de ensayos & $\begin{array}{l}\text { Grado de certeza o de conocimiento en } \\
\text { relación a un evento }\end{array}$ \\
\hline Datos & Aleatorios, muestra representativa & Fijos, son los que hay \\
\hline Parámetros & Fijos & Aleatorios \\
Intervalo de confianza del $k \%$ & $\begin{array}{l}\text { Un intervalo que incluirá el verdadero valor } \\
\text { del parámetro en un } k \% \text { de todas las posi- } \\
\text { bles muestras }\end{array}$ & $\begin{array}{l}\text { Unabilidad del } k \% \text { de incluir el parámetro. Se } \\
\text { llama intervalo de credibilidad }\end{array}$ \\
\hline Conclusión & $P(X / H)\left(\right.$ ie.P(Rechazo $\left.\left.H_{0} / H_{0}=V\right)\right)$ & $P(H / X)$ \\
\hline
\end{tabular}

local en términos de frecuencias de presentación de los posibles diagnósticos. Ambos en conjunto permiten establecer la probabilidad de atribución a priori, antes de conocer la clínica y los exámenes, de los posibles diagnósticos. En segundo lugar es necesario tener un conocimiento acabado de los resultados esperados en cada diagnóstico (conocimiento médico), estableciendo la función de verosimilitud, lo que permite actualizar la distribución de probabilidades de los posibles diagnósticos. El teorema de Bayes permite identificar los pilares diagnósticos relevantes: Probabilidad $a$ priori basada en la epidemiología y la función de verosimilitud, basada en el conocimiento médico (experiencia y ciencia) (Figura 2).

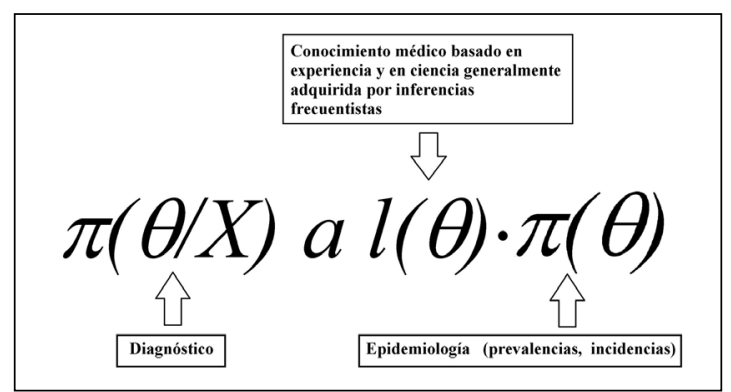

Figura 2. Diagrama conceptual de la generalización del teorema de Bayes. La probabilidad o la distribución de probabilidades de un parámetro $(\theta)$ especificado en una hipótesis (o diagnóstico) dado un conjunto de datos $(\pi(\theta / X))$ es proporcional al producto de la verosimilitud $(\lambda(\theta)=P(X / \theta))$ por la probabilidad o distribución a priori del parámetro $(\pi(\theta))$. Las probabilidades a priori en medicina en general se estipulan basados en la epidemiología, mientras que la verosimilitud depende del conocimiento específico de hechos presentes en cada patología. Esta última depende por una parte de la experiencia individual del médico que hace el diagnóstico y del conocimiento generado por estudios científicos.
Veamos un ejemplo artificial que nos permita ilustrar cómo opera el proceso. Un paciente acude a nuestra consulta y supongamos que tenemos la dicotomía representada en dos hipótesis: $H_{1}=$ patología maligna, $H_{0}=$ patología benigna. $\mathrm{La}$ probabilidad a priori $\mathrm{P}\left(\mathrm{H}_{1}\right)$ corresponde a la prevalencia de neoplasia para un sujeto chileno que la estimaremos aproximadamente $194 / 100.000^{30}$; $P\left(H_{1}\right)=0,00194$, por lo que $O_{0}=0,00194 /$ $(1-0,00194)=0,00194377$. Sin embargo, este paciente acudió a la consulta por algún motivo los que aumenta su $O d d s$, supongamos que el $L R$ de acudir a la consulta es 10 , entonces ahora $O p$ $H_{1}=10 \mathrm{xO}=0,0194377$. Pero el paciente tiene mas de 70 años $(L R=4,16)$, entonces ahora $O p$ $H_{1}=0,0194 \mathrm{x} 4,16=0,0808$, ha tenido hemoptisis $(L R=5,08)$ ahora $O p H_{1}=0,41$, hay historia familiar de malignidad $(L R=4,95)$ ahora $O p H_{1}=2,03$, tiene un nódulo en la radiografía en lóbulo superior $(L R=1,22)$, ahora $\mathrm{OpH}_{1}=2,48$, el nódulo es espiculado $(L R=5,54)$, ahora $O p H_{1}=13,74$, mide más de $3 \mathrm{~cm}(L R=5,23)$, ahora $O p H_{1}=71,87$. Es decir, el paciente inicialmente tenía una $O d d s$ baja de neoplasia pero con los exámenes (datos: $X_{i}$ ) ahora tiene una Odds muy alta. Podemos calcular su probabilidad $P\left(H_{1}\right)=71,87 /(71,87+1)=0,986$ (valores de $L R$ en 31 ). Entonces, ahora podemos afirmar que el paciente tiene un cáncer con una probabilidad de error de 0,013 .

Para algunos lo esencial del enfoque Bayesiano está en el uso de probabilidad como credibilidad subjetiva a priori. Sin embargo, es necesario aclarar que la subjetividad solo yace en la distribución de probabilidades a priori. Así, podemos usar priori's objetivas versus subjetivas e informativas versus no-informativas. Por ejemplo una priori no 
informativa es una distribución uniforme (igual probabilidad en cada diagnóstico) pero puede ser objetiva (basada en datos previos) o bien subjetiva ${ }^{26}$. Habitualmente en medicina usamos la distribución a priori relativamente objetiva e informativa aportada por la realidad epidemiológica local.

La razón de verosimilitudes es el factor potenciador de la Odds a priori. Si el factor de Bayes u odds a posteriori es similar a la Odds a priori, entonces la contribución al diagnóstico que hicieron los datos $\left(X_{i}\right)$ en la hipótesis final es escasa, mientras que si existen grandes diferencias, es el peso de los datos el que permite una adecuada proposición final.

Lo expuesto no significa que el método pueda ser usado directamente, ya que es virtualmente imposible introducir en este algoritmo todas las posibilidades diagnósticas ni calcular todas las verosimilitudes (volvemos a Gödel), las cuales pueden variar en los subtipos de las entidades diagnósticas haciendo que las interfaces en cada paso sean situación-dependiente ${ }^{32}$. Afortunadamente este proceso es mental y no pasa por cálculos numéricos. Así, el proceso diagnóstico no sigue estrictamente el álgebra definida en la inferencia Bayesiana sino que coincide en su estructura general, estableciendo una inferencia inductiva cuasi-Bayesiana que conduce a proposición de una hipótesis con una cierta incerteza, con una metodología científica.

Volviendo a la anécdota inicial, cuando nuestro profesor hacía la pregunta: "La medicina es: a) un arte; b) una ciencia y c) una ciencia y un arte”. Ahora la respuesta es b).

En realidad no. No arruinemos la magia... también es un arte.

\section{Referencias}

1. Norman G, Sherbino J, Dore K, Wood T, Young M, Gaissmaier W, et al. The Etiology of Diagnostic Errors: A Controlled Trial of System 1 Versus System 2 Reasoning. Acad Med 2014; 99 (2): 277-84.

2. Croskerry P, Petrie DA, Reilly JB, Tait G. Deciding About Fast and Slow Decisions. Acad Med 2014; 89: 285-91.

3. Mamede S, Schmidt HG, Rikers RJM, Penaforte J, Coelho J. Breaking down automaticity: Case ambiguity and the shift to reflective approaches in clinical reasoning. Med Educ 2007; 41: 1185-92.
4. Mamede S, Schmidt HG, Rikers RM, Custers EJ, Splinter TA, van Saase JL. Conscious thought beats deliberation without attention in diagnostic decision-making: At least when you are an expert. Psychol Res 2010; 74: 586-92.

5. Croskerry P. The importance of cognitive errors in diagnosis and strategies to minimize them. Acad Med 2003; 78: 775-80.

6. Ilgen JS, Bowen JL, Yarris LM, Fu R, Lowe RA, Eva K. Adjusting our lens: Can developmental differences in diagnostic reasoning be harnessed to improve health professional and trainee assessment? Acad Emerg Med 2011; 18 (suppl 2): S79-S86.

7. Püschel K, Montero J, Chacón M, Peñaloza B. Diagnóstico clínico y toma de decisiones en medicina ambulatoria. Revista Chilena de Medicina Familiar 2017; 1 (5): 5-10, jul. 2017. http://www.tricahuescholar.com/ tricahue/index.php/sochimef/article/view/229

8. Nicoll D, Lu ChM, Pignone M, McPhee SJ. Guia de pruebas para diagnóstico. Sexta edición. México: McGraw-Hill; 2014.

9. García RE. El concepto de calidad y su aplicación en medicina. Rev Med Chile 2001; 129: 822.

10. Crespo L. Kurt Gödel, gigante de la lógica. Fides et ratio 2008; 2: 20-28.

11. Tankard J Jr. The Statistical Pioneers. Cambridge, MA: Schenkman; 1984.

12. Rodríguez E. Estadística y Psicología: Análisis histórico de la inferencia estadística. Perspectivas psicológicas 2005; 15: 96-102.

13. Bunge M. Las pseudociencias. Pamplona, España: Editorial Laetoli; 2010.

14. Steel RGD \& Torrie JH. Bioestadística: Principios Y Procedimientos. Bogotá: McGraw-Hill Latinoamericana SA; 1980.

15. Feller W. An Introduction To Probability Theory And Its Applications. New York: John Wiley \& Sons; 1968.

16. Cramer. Elementos de la Teoría de Probabilidades. Madrid: Aguilar SA; 1996.

17. Colquhoun D. An investigation of the false discovery rate and the misinterpretation of $\mathrm{p}$-values. R. Soc Open Sci 2014; 1: 14026. http://dx.doi.org/10.1098/ rsos. 140216.

18. Zar JH. Biostatistical Análisis. New Yersey: Prentice Hall; 1996.

19. Cohen J. Things I Have Learned (so far). American Psychologist 1990; 45: 1304-12.

20. Cohen, J. The Earth is Round ( $\mathrm{p}<0.05)$. American Psychologist 1994; 49: 997-1003.

21. Kraemer HC, Thiemann S. How Many Subjects? Statistical Power Analysis in Research. Newbury Park, CA: Sage Publications, Inc; 1987. 
22. Murphy KR, Myors B. Statistical Power Analysis. Mahwah, NJ: Lawrence Erlbaum Associates, Publishers; 2004.

23. Wasserstein RL. Lazar NA. ASA statement on statistical significance and p-values. Amer Stat 2016; 70 (2): 12933.

24. Canals M. Bases cuantitativas del análisis de imágenes médicas: Información e inferencia Bayesiana. Rev Med Chile 2003; 131: 553-6.

25. Canals M, Sabbagh E, Chernilo S. Neumonias en el inmunocomprometido: perspectiva desde el diagnóstico por imágenes, e inferencia Bayesiana. Rev Chilena Infectol 2014; 31: 119-33.

26. Bernardo J, Smith AFM. Bayesian Theory. Hoboken, New Jersey: John Wiley \& Sons, Inc; 1994.

27. Lee PM. Bayesian statistics: an introduction. Hoboken,
New Jersey: John Wiley \& Sons, Inc; 2012.

28. Sackett DL, Strauss SS, Richardson WS, Rosenberg W, Haynes RB. Medicina Basada En La Evidencia. Madrid: Harcourt; 2001.

29. Ellison AM. An introduction to Bayesian inference for ecological research and environmental decision-making. Ecol Appl 1996; 6: 1036-46.

30. MINSAL. Primer informe de registros poblacionales de cáncer en Chile. 2012. https://www.paho.org/chi. Consultado 18/1/2019.

31. Gurney JW, Lyddom DM, McKay JA. Determining the likelihood of malignancy in solitary pulmonary nodules with Bayesian analysis. Part II. Application. Radiology 1993; 186: 415-22.

32. Miettinen OS. The modern scientific physician: 3. Scientific diagnosis. JAMC 2001; 165 (6): 781-2. 